\title{
Meaning of the $45^{\circ}$-angle in mechanical design according to nature
}

\author{
C. Mattheck, R. Kappel \& O. Kraft \\ Institute for Materials Research II, \\ Forschungszentrum Karlsruhe, Germany
}

\begin{abstract}
In nature, evolution forces plants and animals to most efficiently use energy and material. Their mechanical load carrying capability depends on material properties, on the shape and constructive setup as well as on the failure mechanism of the complete structure. The mechanical frames of biological structures typically consist of fibrous composite where the fibres often can be interpreted as tension ropes.

Tension-loaded components are highly qualified, especially for materialefficient and lightweight structures. The advantage of tension-loaded components is that they cannot fail by kinking or buckling if their aspect ratio is too high, unlike pressure-loaded components. However, plants and animals do not only resist pure tension loads but also compression, and shear loads are often absorbed by a tricky design of shape and material.

The present paper will analyze various natural structures and explain their design principle. It will present design rules of nature using the $45^{\circ}$-angle based on the "Shear-Square" and the "Method of Tension Triangles". By use of these methods ultra light and durable technical components can also be designed according to nature without the use of the Finite Element method.
\end{abstract}

Keywords: Thinking in Ropes, Shear-Square, Method of Tensile Triangles lightweight design, biological design, optimization of components.

\section{Introduction}

In nature, one of the features evolution performs most efficiently is creating lightweight designs by adaptation. The saving of weight realizes the economization of finite resources such as material and energy as a benefit for survival. 
Apart from the absolute weight the combination of load, material and design is most significant for the sustainability of lightweight design. To resist the operating load, fibre-reinforced composites are established as building materials for the load bearing structures of plants and animals.

The mechanical properties of those biological materials, especially of the fibres, are jointly responsible for the design of biological load carriers. On closer examination similarities in design for different biological structures and materials appear. The presence of the $45^{\circ}$-angle in shape and structure becomes more and more obvious.

\section{Methods}

\subsection{The "Shear-Square"}

The square formation of shear stresses that is common in mechanical literature can also be used to visualize the force flow [1]. The descriptive approach of a small rotatable square "nailed" on the slip line of the shear shows that it would turn under longitudinal shear stress (Fig 1a). As imaginary shear-squares do not rotate inside the component, counteracting transverse shear stresses of equal magnitude avoid the rotation (Fig. 1b). Tensile and compressive stresses displaced by $45^{\circ}$ are equivalent to these shear stresses (Fig. 1c). This simple but effective tool does not substitute any stress calculations but it gives a good view of the local force flow and supports the mindset for lightweight design "Thinking in Ropes" [2].

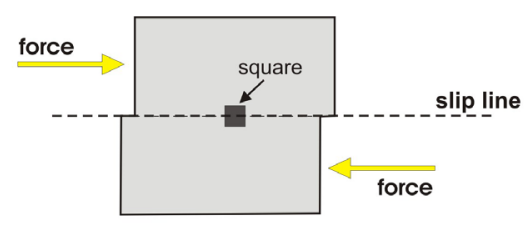

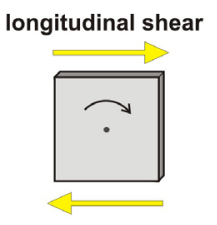

(A)

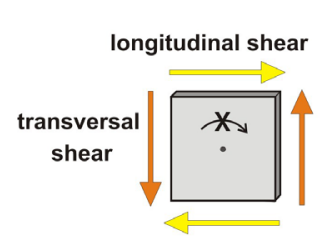

(B)

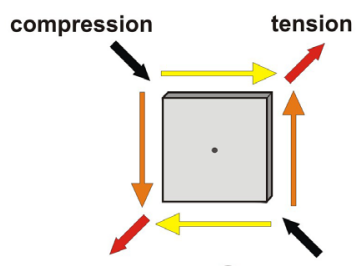

(C)

Figure 1: Non-rotating shear-square and equivalent tension and compression stresses.

\subsection{The "Method of Tensile Triangles" (MTT)}

The Method of Tensile Triangles is based on the design rules of nature. It provides the reduction of notch stresses as well as the removal of underloaded parts in mechanical components [3]. 
A major mechanical design rule of nature, the "axiom of uniform stress" aims to distribute stress homogeneously in a structure. Due to the disturbance of force flow at a sharp kinked angle, notch stresses will occur, leading to an inhomogeneous stress distribution along the contour. Those notch stresses could also be explained by use of the Shear-Square [4]. Looking at the rectangular edge in Fig. 2a under tension loading, we can identify the direction of tension stresses resulting in the longitudinal shear along the potential slip line. To avoid stress peaks, the shoulders may be bridged by a supporting rope (Fig. 2b). The substitution of the rope with a "tension triangle" results in a new contour with two less dangerous notches of larger angles (Fig. 2c).

For a uniaxial stress state the more dangerous notch can also be bridged by an isosceles tensile triangle starting at the centre of the hypotenuse of the previous triangle. The repetition of this procedure is the Method of Tensile Triangles (Fig 3, left). In a last step, the segments are rounded to obtain an optimized notch design. The new contour line follows the local force flow and also separates the required material from the needless material.

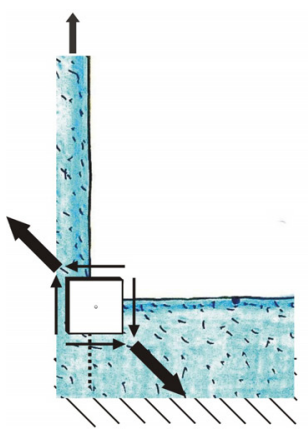

Figure 2:

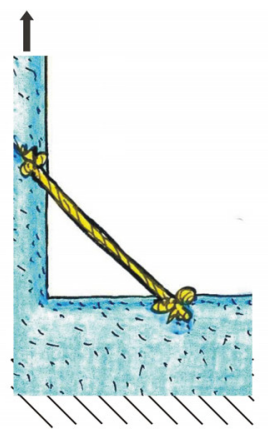

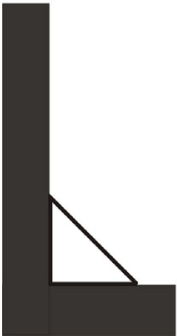

(1)

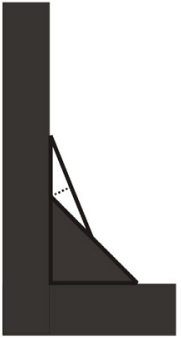

(2)

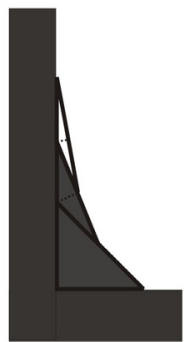

(3)

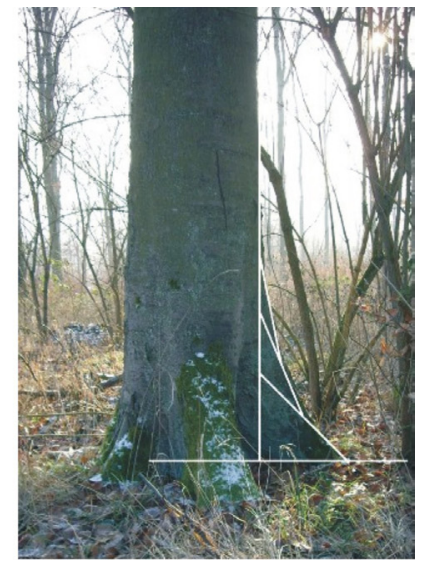

Figure 3: $\quad$ Method of Tensile Triangles. 
The application of the MTT to the shape of plants and animals gives a good analogy to natural design [5]. Figure 3 shows the re-design of the transition at the foot of a tree's trunk to the broader root plate at the windward side.

\section{$3 \quad 45^{\circ}$-angle applied in biological design}

The basic materials of biological supporting structures are fibre-reinforced composites. From the mechanical point of view, various body structures could be explained by using the "Shear-Square" and "Method of Tensile Triangles" tools. The load setting in combination with the fibrous material determines the design and vice versa. In case of shear loading, the $45^{\circ}$-angle plays an important role [6].

\subsubsection{Thorns and spikes of plants}

Thorns and spikes defend the plants from herbivores. Like the plants themselves they are built of wooden material including cellulose fibres. For sustainable protection not only the tip is painfully spiky, but also the transition at the base is highly optimized to avoid abruption. The design of the transition from the thorn to the plant looks similar to the shape shown in Figure 3. This design can be observed for different species with variations depending on the slenderness of the thorn. Using the Shear-Square as shown in Figure 2a at the base of the thorn, the appearance of a $45^{\circ}$-angle at the base of the thorn becomes comprehensible.
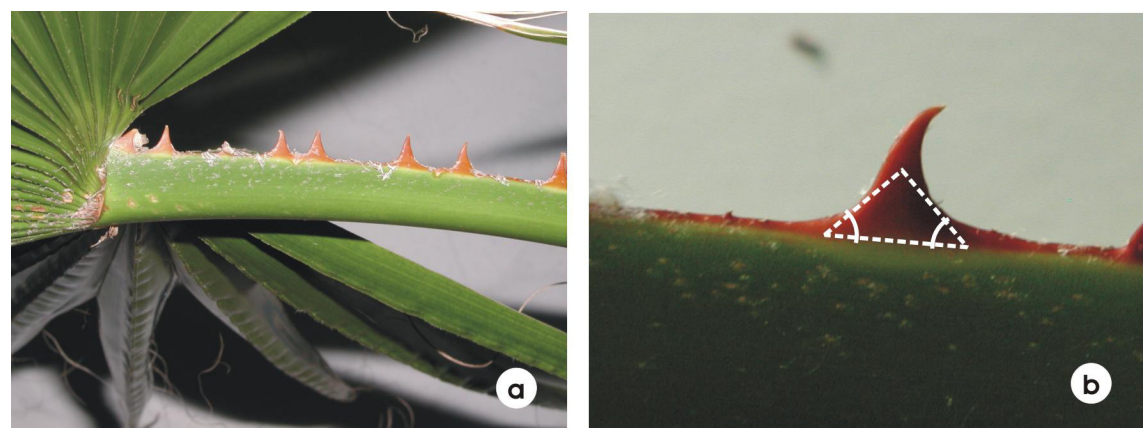

Figure 4: Palm tree with thorns along its petiole.

The vast majority of palm trees (Arecaceae) live in the tropics. Palms are abundant throughout the tropical regions around the world, and are present in almost every type of habitat. Many species are very well adapted to the aridity and high temperatures of the dessert. The growth habit of palms is usually a straight, unbranched stem with large evergreen leaves, which are spirally arranged at the top of the stem. For some species the leaves are protected with thorns along their petioles against herbivores. In Figure 4 a symmetrical triangle with two $45^{\circ}$-angles at the base of the thorn is plotted, while the tip of the thorn is directed against the stem. 
Water lilies (Nymphaeaceae) are floating leaf plants. They are rooted in soil in bodies of water. Due to the scarceness of oxygen at the ground level, their leaves are essential respiratory organs. Floating on the water surface, they are attached to the root with long flexible petioles for exchange. The upper side of the leaves, including the stomata, is protected against wetting by a waxy layer. In addition almost symmetrical thorns like a defensive fortification shield the round leaf with the $45^{\circ}$-angle at the jagged leaf edge (Figure 5).

\subsection{Exoskeletons of arthropods}

More than $80 \%$ of living animal species are arthropods including insects, arachnids and crustaceans. Arthropods are covered by a hard exoskeleton made of fibrous chitin, a polysaccharide, which provides physical protection and resistance to desiccation. Because it cannot be adapted over a period of growth, an arthropod periodically sheds this covering when it moults.

The largest known living arthropod is the Japanese spider crab (Macrocheira kaempferi), with a leg span up to almost 4 meters. Its habitat is limited to the Pacific side of the Japanese archipelago, where they live on the seabed at depths of 50 down to 300 meters. With the huge toothed claws at the anterior limbs they crush mussels and other crustaceans for nutrition. Although the claws are built of a thin layer they are able to apply immense forces between the fixed and a movable part. At the junction of the fixed part the $45^{\circ}$-angle appears (Fig. 6) [6].

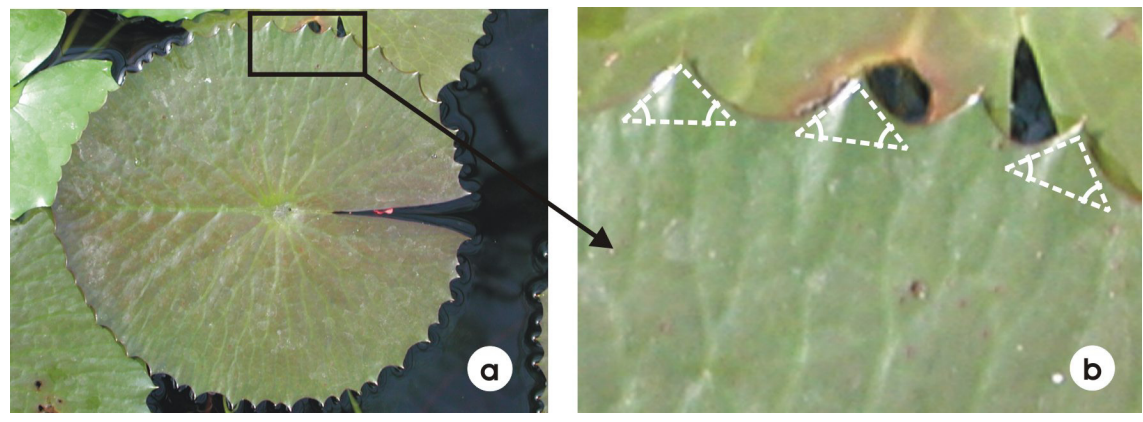

Figure 5: $\quad$ Spiky leaves of a water lily.
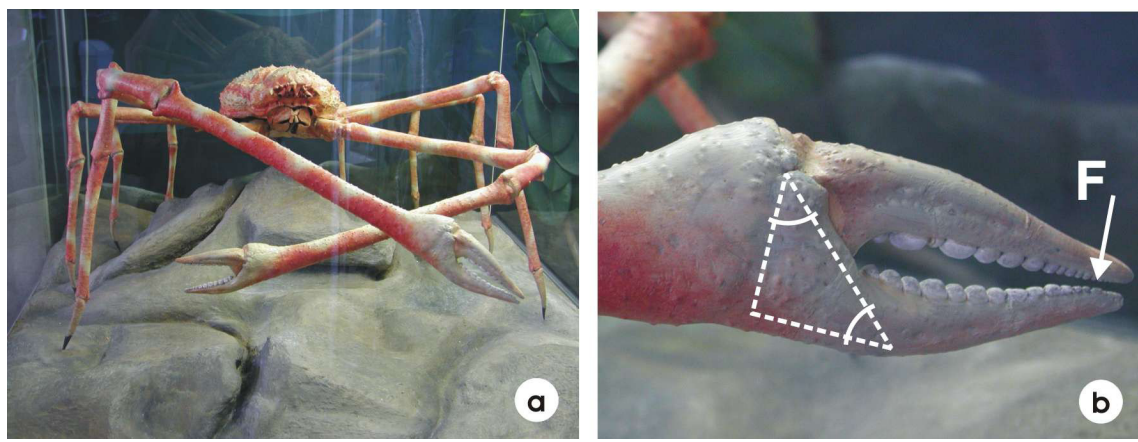

Figure 6: Japanese spider crab (Macrocheira kaempferi). 

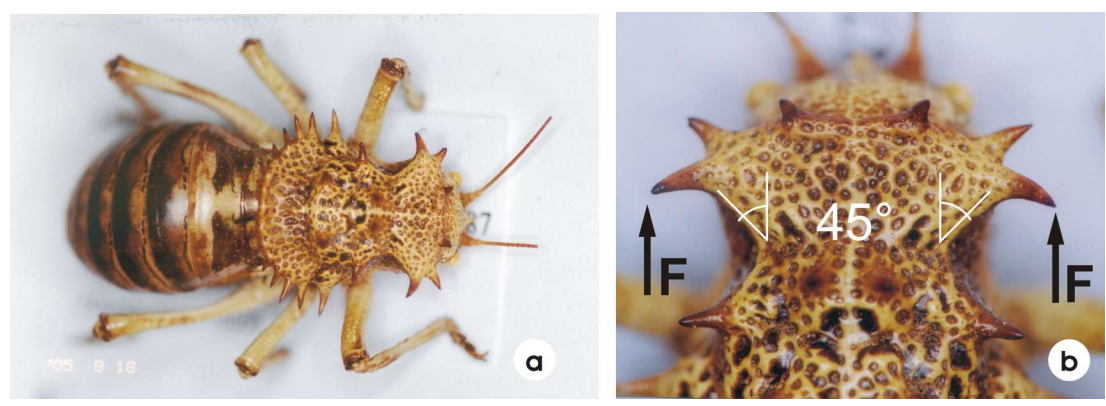

Figure 7: Armoured insect with spiky designed exoskeleton.
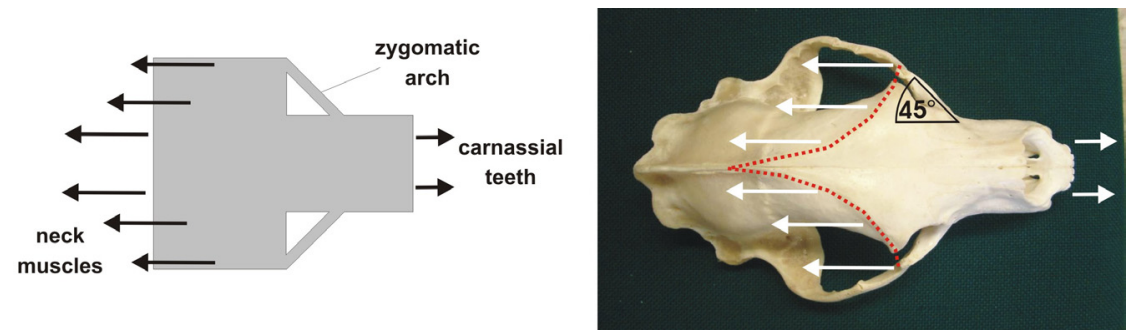

Figure 8: $\quad$ Schematic of a predator skull in comparison to a black bear skull.

The spiky head shield of an armoured insect is shown in Figure 7. The design of the intersections between the spikes also offers a mechanically reasonable $45^{\circ}$-angle modelling the thin shell.

\subsection{Endoskeletons of mammals}

The endoskeleton of mammals is an accumulation of rigid bones, which are primarily comprised of collagen fibres and minerals. Bones are highly loaded in extreme situations. To reduce failure, the material properties of bones are optimized as well as their design. The skull of mammalian predators is a good example of an optimized shape. For an unfavourable weight ratio between prey and predator the interaction requires a strong chain of links to transmit the forces from the carnassial teeth via the skeleton downwards into the ground. Therefore, the filigree zygomatic arches act like supporting ropes to reduce shear stresses at the transmission from snoot to neck as shown schematically in Figure 8 and exemplified by the skull of a black bear [6].

Warthogs are omnivores, snuggling the ground with their giant bizarre head. The length of their upper tusks may achieve up to 60 centimetres. The lower tusks are razor-sharp weapons. The dangerous tusks of the warthogs are fixed to the skull at a $45^{\circ}$-angle. The broadening of the skull from snoot to neck also shows a $45^{\circ}$-angle (Figure 9). The triangles plotted in the photo on the right-hand side are similar to the first Tensile Triangle in the optimization of the notch form. Interestingly, the shape of the head is quite similar to the head of an insect in Figure 6. 

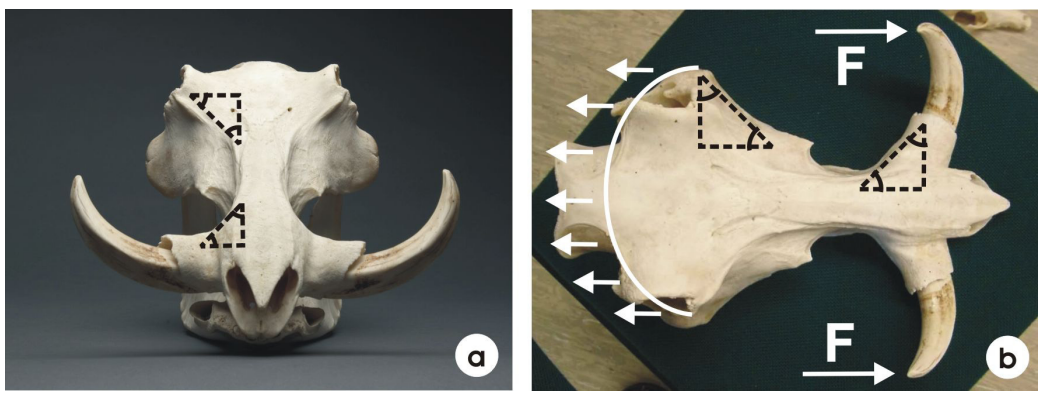

Figure 9: $\quad$ Skull of a warthog with tusks.
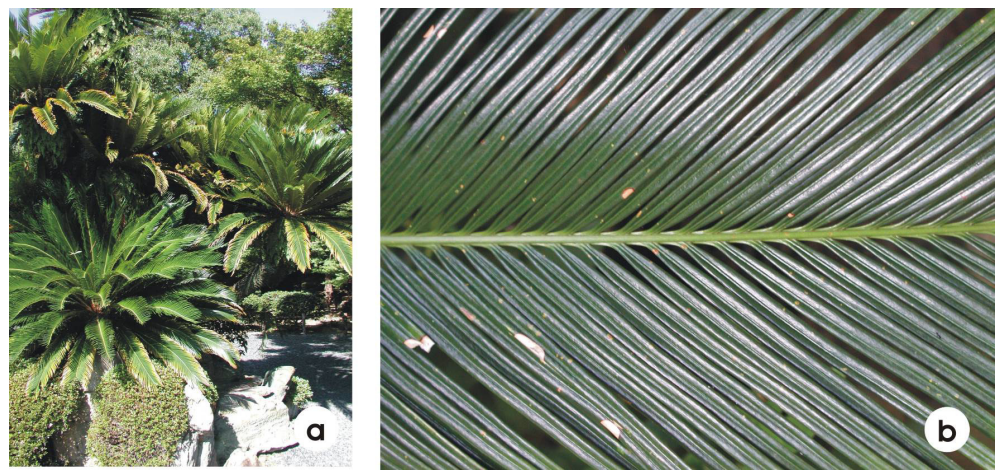

Figure 10: Feather-leaved palm tree.

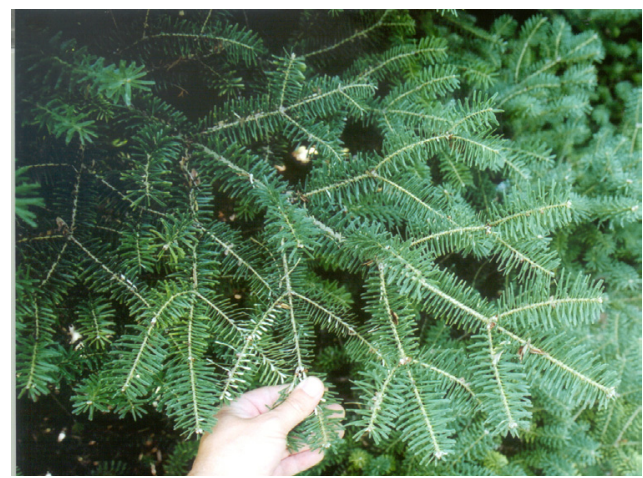

Figure 11: $\quad$ Iterative branching pattern of a conifer (Abies koreana Wils).

\subsection{Frameworks}

To reduce shear load between adjoining structures the appearance of $45^{\circ}$-angles is common in nature. The out branching under $45^{\circ}$ shows a pattern similar to a fishbone, where shear between the vertebrae and remaining fish occurs during acceleration. This pattern composes a framework that also enables mechanical effective support between the featherlike leaves of a plant (Figure 10). 
For the conifer shown in figure 11 , the pattern is repeated iterative using $45^{\circ}$ angles. In this way the economization of material of a lightweight structure is increased; each structure is stable at service load and in addition supported by its neighbours for higher impacts.

\section{Conclusion}

Lightweight design and its sustainability are controlled by the combination of material, load and shape. In nature biological supporting structures are built of fibre composite material. Acting like small tension ropes the fibres are perfect lightweight structures. Their arrangements according to the force flow cause the design.

Thorns and spikes of different species, plants and animals, show a remarkable affinity in their design. From the mechanical point of view they are like cantilever beams with an optimized transition at the base using the $45^{\circ}$-angle. Notch stresses will arise from an abrupt deviation of the force flow. The given examples demonstrate the omnipresence of the $45^{\circ}$-angle for reducing those notch stresses. The smoothed edges could be redesigned by use of the Method of Tensile Triangles.

A feather like branching using the $45^{\circ}$-angle is a mechanical optimized interface between neighbouring structures loaded by shear. Together they generate a mechanical community defending each other from impacts beyond individual service load.

The $45^{\circ}$-angle seems to be a mechanical constant for load adaptive lightweight design of biological load carriers. It enables conversion of critical shear load into adequate tension load or if necessary compression load. To transfer these biological design rules into the engineering design of lightweight structures, the mindset "Thinking in Ropes" is proposed with the "Shear-Square" and the "Method of Tensile Triangles" as useful and simple building blocks.

\section{References}

[1] Mattheck, C., Bethge, K., Ein Denkwerkzeug - Die Methode der Schubvierecke. Konstruktionspraxis 3, (2007), 32-34.

[2] Mattheck, C., Kappel, R., Tesari, I., Kraft, O., In Seilen denken - Einfache Anleitung fuer naturnahes Konstruieren. Konstruktionspraxis 9, (2004), 2629.

[3] Mattheck, C., Kappel, R., Sauer, A., Shape optimization the easy way: The "Method of Tensile Triangles". Int. Journal of Design \& Nature. Vol. 2, No. 4(2007), 1-9.

[4] Mattheck C., The face of failure - in nature and engineering, Verlag Forschungszentrum Karlsruhe GmbH (2004).

[5] Mattheck, C., Secret design rules of nature - Optimum shapes without computers. Verlag Forschungszentrum Karlsruhe GmbH (2007).

[6] Kappel, R., Zugseile in der Natur. FZKA 7313, Verlag Forschungszentrum Karlsruhe GmbH (2007). 